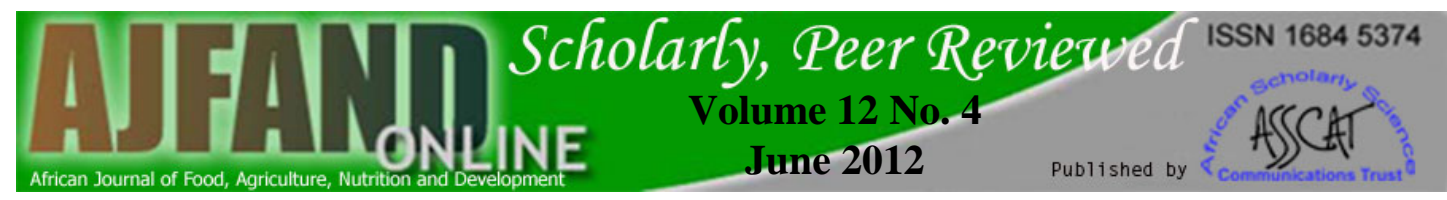

\title{
STRENGTHENING FOODBORNE DISEASES SURVEILLANCE IN THE WHO AFRICAN REGION: AN ESSENTIAL NEED FOR DISEASE CONTROL AND FOOD SAFETY ASSURANCE
}

Mensah $\mathbf{P}^{1^{*}}$, Mwamakamba $\mathrm{L}^{1}$, Kariuki $\mathrm{S}^{2}$, Fonkoua $\mathrm{MC}^{3}$ and A Aidara-Kane ${ }^{4}$

*Corresponding author email: $\underline{\text { mensahp@afro.who.int }}$

${ }^{1}$ World Health Organization Regional Office for Africa, BP 06, Brazzaville, Republic of Congo.

${ }^{2}$ Chief Research Officer, and Head, Centre for Microbiology Research, Nairobi, Kenya.

${ }^{3}$ Senior Microbiologist, Head of Laboratory, Laboratory of Bacteriology, Centre Pasteur du Cameroun, Yaoundé, Cameroon.

${ }^{4}$ World Health Organization, Headquarters. 


\section{ABSTRACT}

Several devastating outbreaks of foodborne diseases have been reported in the African region including acute aflatoxicosis in Kenya in 2004 and bromide poisoning in Angola in 2007. There are concerns about transmission of multiple antibiotic resistant bacteria and pesticide residues in foods. The globalization of the food trade which could increase the spread of food contaminants internationally is an emerging issue. The new International Health Regulations (IHR) (2005) cover events of international importance including contaminated food and outbreaks of foodborne disease. The IHR (2005) and other international as well as regional agreements require Member States to strengthen surveillance systems including surveillance for foodborne diseases. WHO has been supporting countries to strengthen foodborne disease surveillance since 2003. This paper reports on the work of WHO and partners in the area of foodborne disease surveillance, the challenges and opportunities and provides perspectives for the area of its work. The paper shows that laboratory-based surveillance is the preferred system for foodborne disease surveillance since it allows early detection of outbreak strains and identification of risk factors with laboratory services as the cornerstone. Foodborne disease surveillance has been included in the revised Integrated Disease Surveillance and Response (IDSR) Strategy and there are guidelines for use by countries. WHO in collaboration with partners, especially the Global Food Infections Network (GFN), has been supporting countries to strengthen national analytical capacity for foodborne disease surveillance and research. Training for countries to detect, control and prevent foodborne and other enteric infections from farm to table has been conducted. The training for microbiologists and epidemiologists from public health, veterinary and food sectors involved in isolation, identification and typing of Salmonella sp, Campylobacter sp., Vibrio cholerae, Vibrio sp. and Shigella from human and food samples have been carried out. Research into specific topics in microbiology and chemical contaminants has been conducted. Three institutions in Cameroun, Mali and Nigeria have been designated as centres of excellence for chemical contaminants. Despite these significant achievements, a number of challenges remain. Most food safety programmes and food safety systems remain fragmented resulting in duplication of efforts and inefficient use of resources; and most laboratories in the African Region are poorly resourced. In countries where facilities exist, there is underutilization and lack of synergy among laboratories. Countries should, therefore, conduct audits of existing laboratories to determine their strengths and weaknesses and strategize as appropriate. It is also imperative to continue to strengthen partnerships and forge new ones and increase resources for food safety, in general, and for foodborne disease surveillance, in particular, and continue capacity building, both human and institutional.

Key words: Foodborne diseases, surveillance, laboratory-based 


\section{INTRODUCTION}

Food, if not handled hygienically, could be a mode for transmission of microbial, chemical and physical hazards. There is concern about transmission of multiple antimicrobial resistant bacteria via the food chain through the use of antibiotics in animal production. As reported in the paper entitled, "Public Health and Food Safety in the WHO African Region", several devastating outbreaks of foodborne diseases have been reported in the African region, including acute aflatoxicosis in Kenya in 2004, that was attributed to with maize [1, 2] and bromide poisoning in Angola in 2007 [3] associated with the use of sodium bromide as cooking salt. These and other outbreaks were detected through the National Integrated Disease Surveillance and Response systems as well as the vigilance of various health sector workers. Active surveillance and investigation of these outbreaks triggered the need to identify the aetiologies, routes for transmission and risk factors in order to find appropriate control measures.

The globalization of the food trade has increased the likelihood of international incidents involving contaminated food through travel, migration and distribution of food. It has, therefore, become necessary to strengthen systems which will allow the early detection, management and prevention of spread of foodborne disease outbreaks. The International Health Regulations (2005) (IHR) [4] came into force on June 15, 2007 and are based on a risk assessment approach. The IHR (2005) cover events of international importance that involve contaminated food and outbreaks of foodborne disease. It requires Member States to strengthen surveillance systems and notify WHO on outbreaks of diseases of international concern including foodborne diseases.

Surveillance is defined as the systematic ongoing, collection, analysis, interpretation, and dissemination of data for public health action (Figure 1) [5]. Its objectives are to estimate burden of disease and the size of the problem; monitor trends and determine whether the situation is getting better or worse; detect outbreaks to determine if urgent action is needed; assess control programs to obtain information on performance; and generate data for risk analysis.

There are four categories of foodborne disease surveillance, namely, no formal system, syndromic surveillance, laboratory-based surveillance and integrated food chain surveillance. Laboratory-based surveillance is the preferred system for foodborne disease surveillance since it allows early detection of outbreak strains and identification of risk factors.

This paper discusses the importance of surveillance in food control and disease prevention, the activities being carried in the WHO African Region in collaboration with countries and partners, identifies the challenges and opportunities for strengthening foodborne disease surveillance and provides some thoughts on future actions to strengthen systems. 


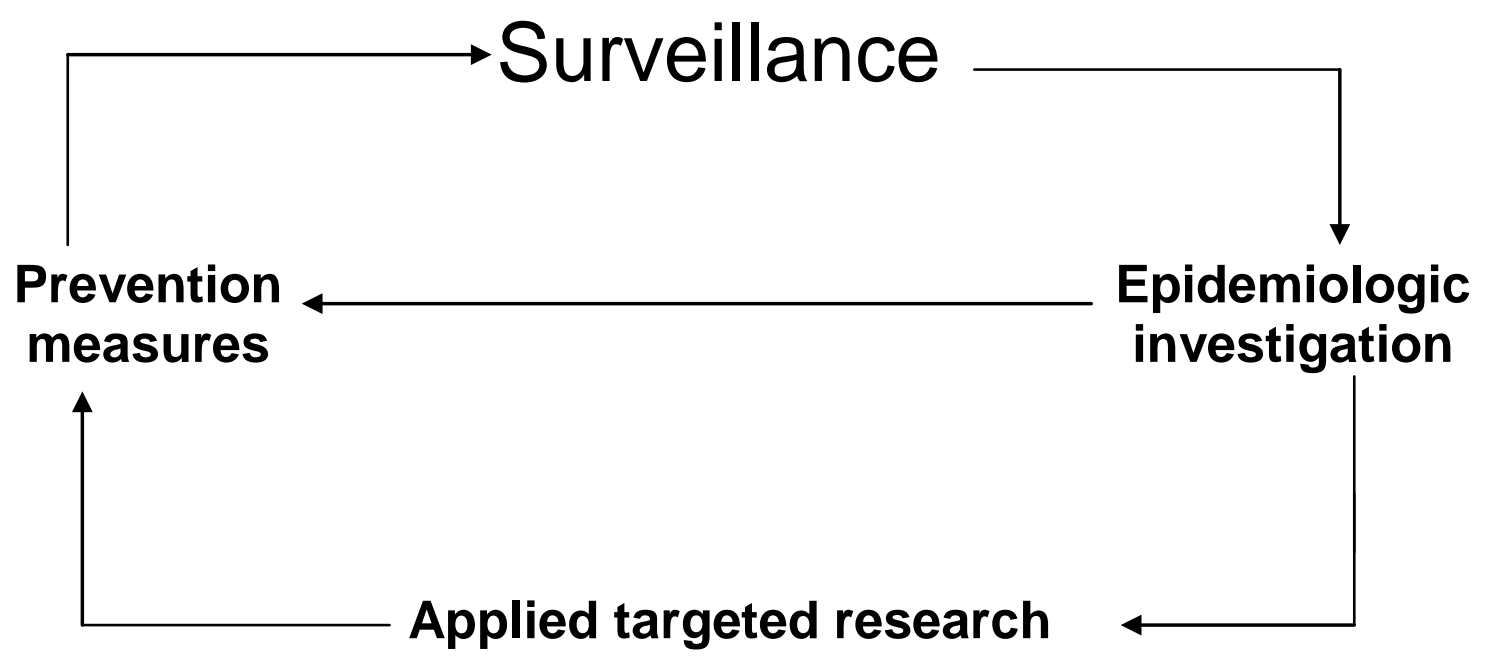

Figure 1: Surveillance drives the cycle of public health prevention

Source: [5]

\section{Laboratory based Foodborne Disease Surveillance}

Laboratory-based surveillance is the systematic ongoing collection, analysis, interpretation and dissemination of data on laboratory-confirmed infections for public health action. The surveillance system uses standard case definitions for classifying diseases. Clinical laboratories are key sources of isolates for laboratory-based surveillance and should routinely send randomly selected isolates to Reference Laboratories for confirmation or subtyping (Figure 2).

Laboratory-based foodborne disease surveillance allows aetiologic identification; determines which pathogens are causing illness or allows aetiologic agent-specific case counts; detects and investigate outbreaks; allows monitoring of trends by pathogen over time and in selected populations; helps to set priorities for control programs; facilitates the evaluation of effectiveness of control programmes; and determines which subtypes of pathogens are causing illness. The information expected includes aetiologic agent-specific trends over time and seasonal variation, definition of at-risk and high-risk populations and recognition of point source of local and diffuse outbreaks at national level.

Sub-typing is important as it provides clues about source and a way to distinguish strains to help detect outbreaks. The key sub-typing methods include sero-typing, Phage-typing, Antibiotic resistance profiling, and pulsed-field gel electrophoresis (PFGE). For example, Salmonella, a common cause of foodborne disease has over 2,500 sero-types with each sero-type having individual biology and epidemiology. 


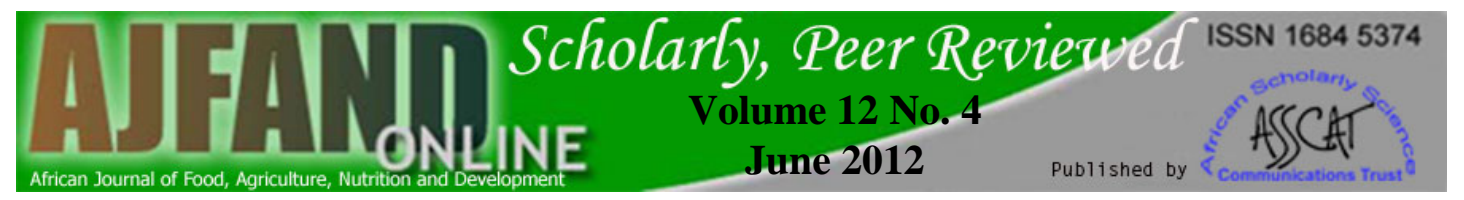

Sero-type Typhi causes typhoid fever, serotype Enteritidis is commonly transmitted by eggs and serotype Typhimurium is transmitted by a wide variety of foods of animal origin.

This surveillance system provides higher quality data than syndromic surveillance and countries are urged to make resources available for its development. Figure 3 illustrates a typical example of laboratory-based foodborne disease surveillance system courtesy of Ministry of Health, Uganda. Laboratory services form part of national epidemic alert and response including detection investigation and response. Laboratory analysis of human, food and animal samples is essential, requiring collaboration with all stakeholders. This must be based on reliable sample collection, transportation, domestic diagnostic capacity and use of outside capacity as required. 

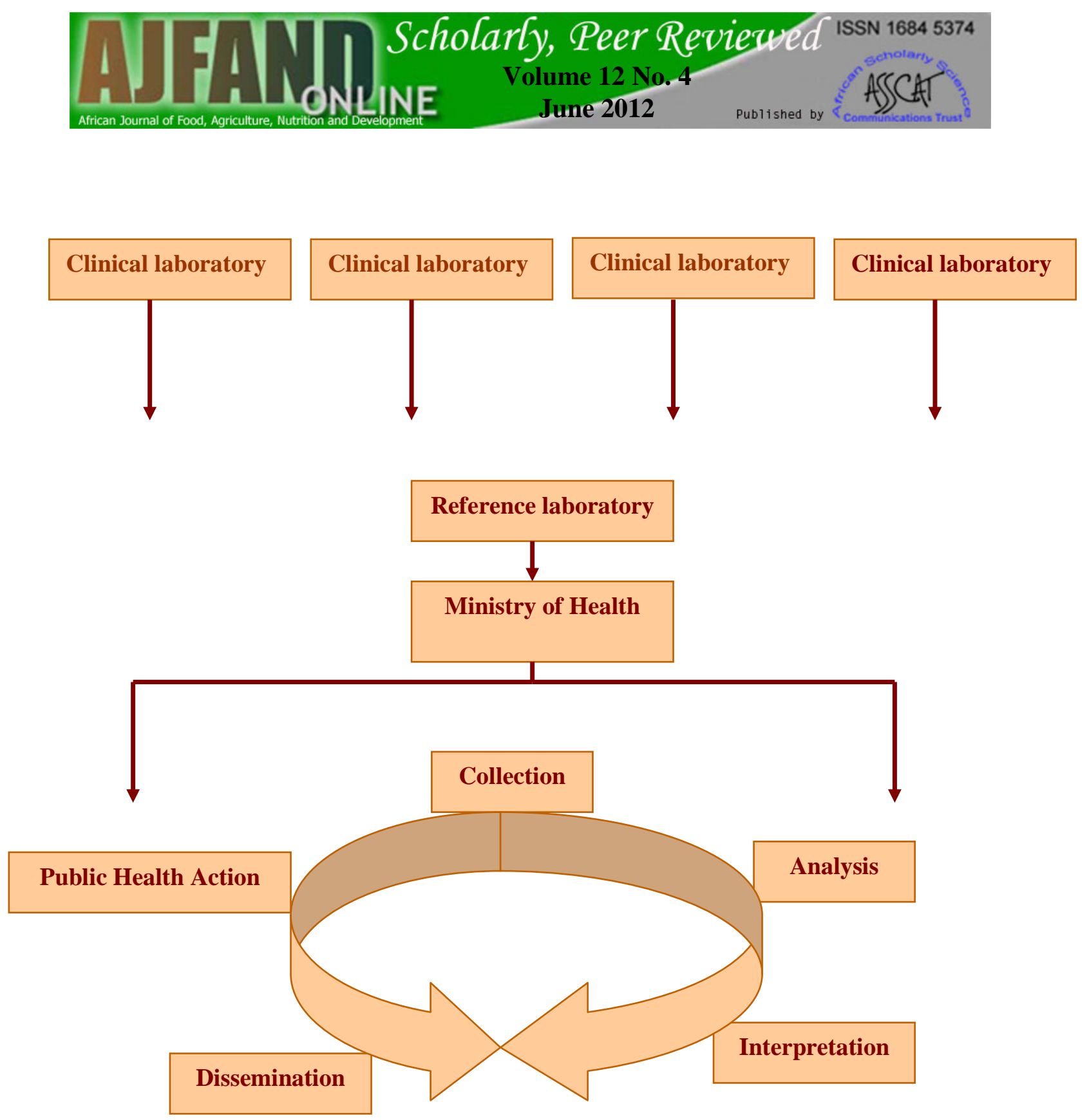

\section{Figure 2: Laboratory-based surveillance}

Source: Presentation by Patricia Griffin (MD), Division of Foodborne, Bacterial, and Mycotic Diseases National Center for Zoonotic, Vectorborne, and Enteric Diseases Centers for Disease Control and Prevention, Atlanta, Georgia, USA (CDC) at the WHO Global Food Infections Network Level II (formerly Global Salm surv ) training, January 2099, Nairobi, Kenya 


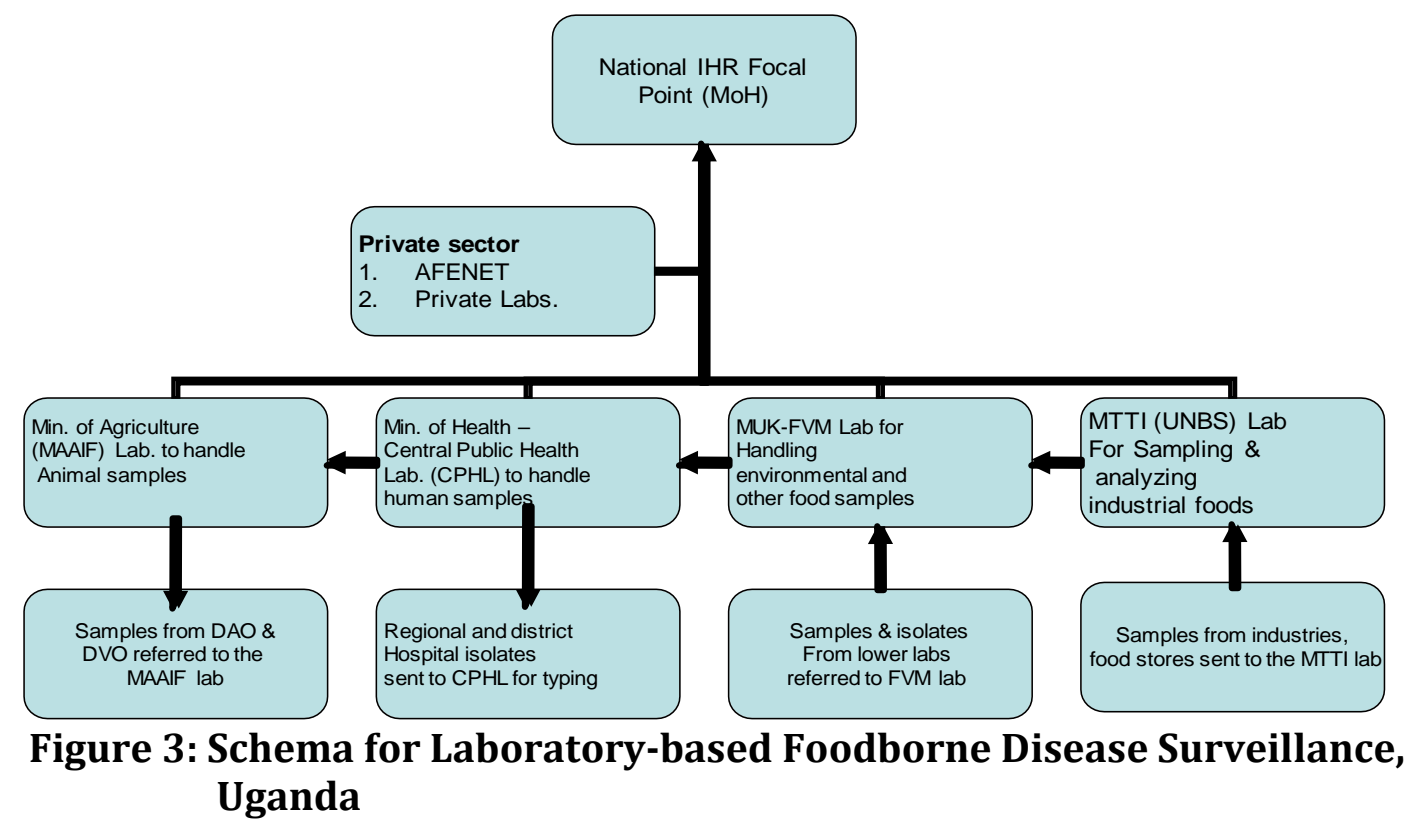

Source: Courtesy Dr Joseph Francis Wamala et al, Epidemiology and Surveillance Division, Department of National Disease Control, Ministry of Health, Uganda

\section{Strengthening foodborne disease surveillance in the WHO African Region}

The document entitled "Food Safety and Health: A Strategy for the WHO African Region (AFR/RC57/4) and resolution AFR/RC57/R2 adopted in 2007 urged Member States to strengthen national analytical capacity for foodborne disease surveillance and research with WHO support. Document AFR/RC59/11 on Policy Orientations on The Establishment Of Centres Of Excellence for Disease Surveillance, Public Health Laboratories, Food and Medicines Regulation including resolution AFR/RC59/R4 adopted in 2009 made similar calls. So far three Centres of Excellence have been established in Cameroun, Mali and Nigeria to analyze chemical contaminants in foods. In 1998, the WHO Regional Office in collaboration with partners established the Integrated Disease Surveillance and Response (IDSR) in the African Region. This horizontal system has established stronger systems linked to laboratory support, resulting in efficient sharing of resources for core surveillance and support functions. The priority diseases addressed by IDSR include cholera, bloody diarrhoea and diarrhoea with dehydration in under-fives. The IDSR investigates the sources of enteric infections including food sources when there are outbreaks but does not require food-related incidences to be reported as required under the IHR 2005. The revised IDSR Strategy includes indicators for foodborne diseases as well the WHO Five Keys to Safer Food as a risk communication tool. 
The WHO Global Food Infections Network (GFN) formerly Global Salm-surv (GSS) initiative have been conducting capacity building activities aimed at improving laboratory-based foodborne disease surveillance since 2002. The mission of GFN is to reduce the global burden of foodborne and other enteric infections by enhancing laboratory-based surveillance and outbreak detection and response; and foster collaboration and communication between microbiologists and epidemiologists working in human health, veterinary and food sciences. GFN collaborates with internationally recognized veterinary, food and public health organizations which provide expertise and guidance. The key activities of GFN include:

(i) External Quality Assurance System (EQAS): assesses the quality of sero-typing and antimicrobial susceptibility testing of Salmonella and other foodborne pathogens in laboratories worldwide;

(ii) Country Databank (CDB): passive surveillance system that collects annual Salmonella summary data from member institutions;

(iii) Reference Testing: technical advice and free of charge reference testing of Salmonella and other enteric pathogens by GFN steering committee partners and regional centres of excellence;

(iv) Electronic Discussion Group (EDG): an electronic mailing list that distributes messages on training materials, articles on foodborne and other enteric infections, and programmatic issues;

(v) Focused regional and national projects: promote continued development and application of techniques learned at GFN International Training Courses.

The programme has provided training for countries to detect, control and prevent foodborne and other enteric infections from farm to table. So far 11 training courses have been held; five in Cameroon, three in Kenya, two in Madagascar and one in South Africa for participants from 37 African countries (Figure 4). Training offered by the GFN includes hands-on practical training in foodborne and zoonotic pathogens, outbreak detection and response for microbiologists and epidemiologists from veterinary and public health institutions (Figure 5). The training involved isolation, identification and typing of Salmonella sp, Campylobacter sp., Vibrio cholerae, Vibrio non-cholerae and Shigella from human and food samples.

WHO is also involved in monitoring antimicrobial resistance (AMR) and has developed Global Principles for Containment of Antimicrobial Resistance originating from use of antimicrobials in livestock production; and reduction of the risk of dissemination of antimicrobial resistant pathogens from animals to humans. Interventions are in the areas of antimicrobial production, distribution and sales; antimicrobial use in animals and its monitoring; strengthening of surveillance of AMR; and improvement of hygiene in food production. 

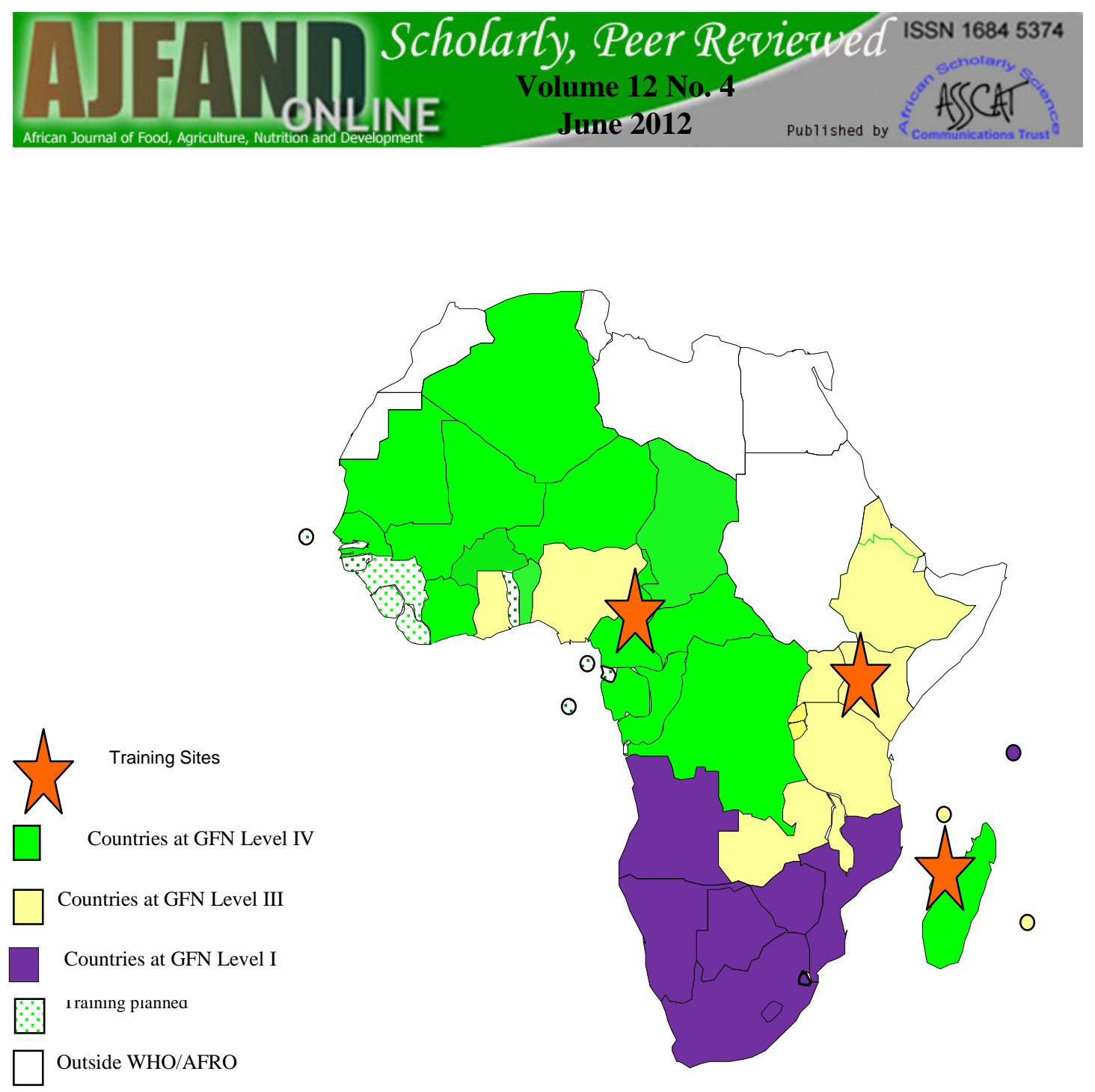

Figure 4: Participating countries and training sites for foodborne disease surveillance 

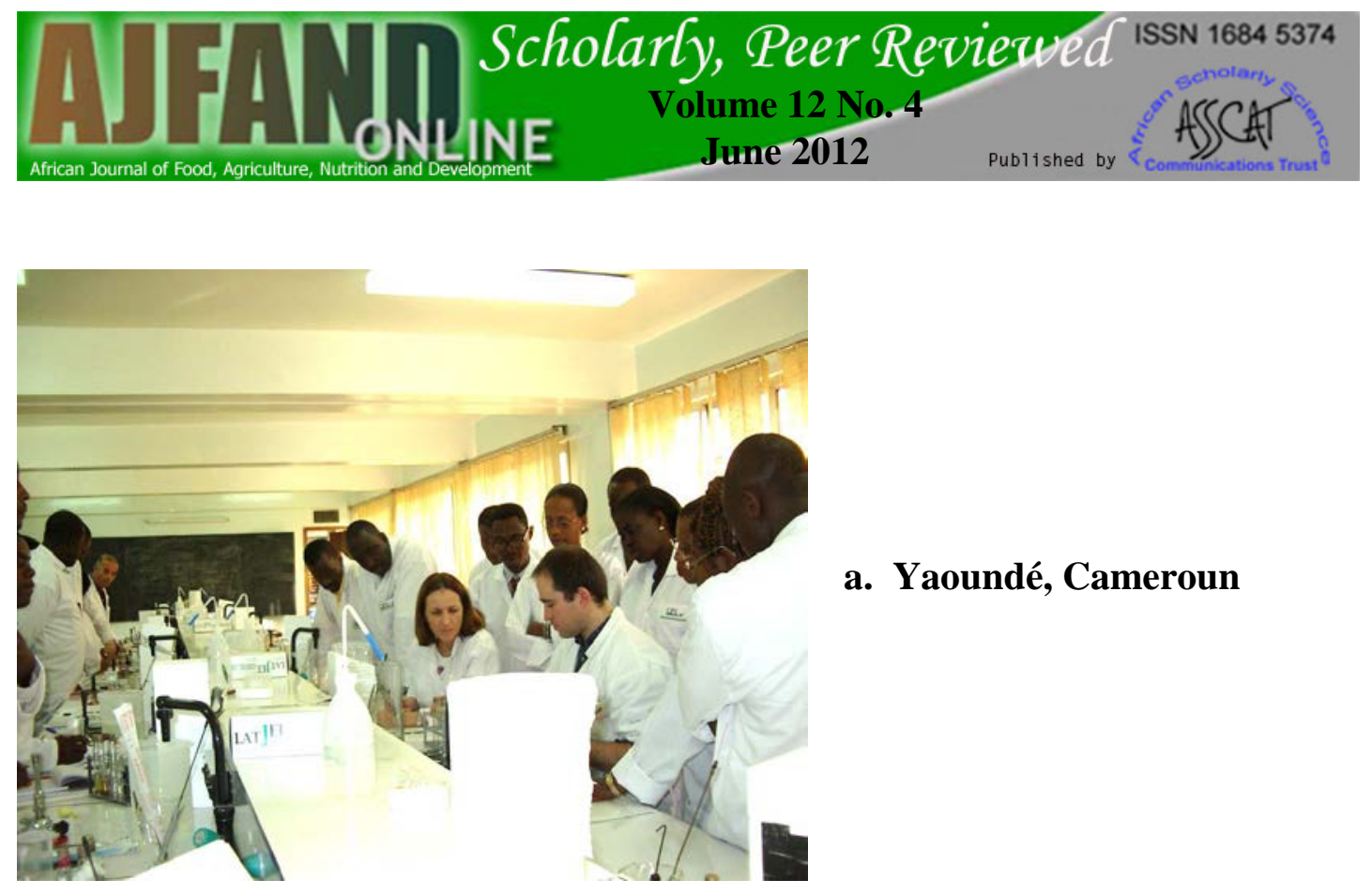

a. Yaoundé, Cameroun

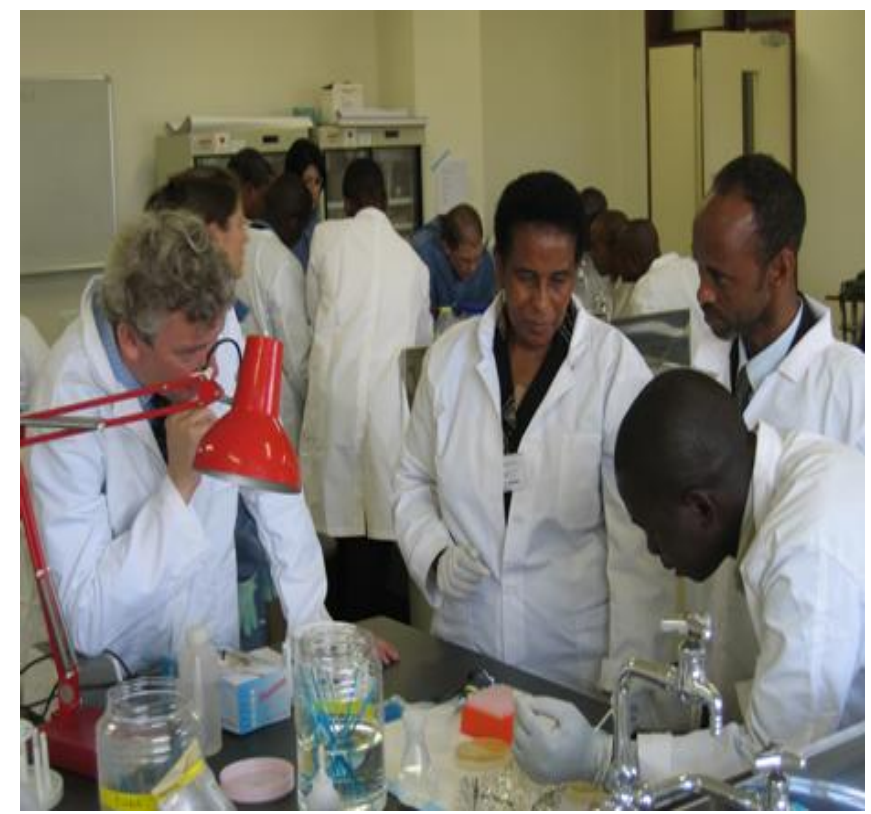

b. Nairobi, Kenya

Figure 5: Training Sessions in two Centres

As part of its capacity-building activities, WHO and its GFN partners, engage in national and regional projects on issues related to food safety and zoonosis through coordination and technical support for diagnostic work, epidemiological analysis, and assistance in publishing results. Box 1 summarises activities being carried at Pasteur Centre, Cameroon. GFN Projects have been performed in Mauritius, Democratic Republic of the Congo, Nigeria and Ethiopia. 


\section{Box 1: Laboratory-based foodborne disease surveillance activities by Pasteur Center of Cameroon}

Pasteur Center of Cameroon (PCC) is the first GFN French speaking training site in African Region. Since 2002, PCC has organized four training courses and two workshops. These included annual training courses concerning isolation, identification and antimicrobial susceptibility testing for Salmonella, Campylobacter, Vibrio cholerae, Shigella from 2002 to 2005; two workshops on "Integrated surveillance of antimicrobial resistance of enteric bacteria" in 2008 and "Strengthening the public health and veterinary national laboratories network for surveillance of foodborne infections” in 2011.

In March 2008, PCC was designated a WHO GFN Regional Centre with additional responsibilities to lead regional communication in foodborne disease and pathogen surveillance, and co-ordinate region-relevant research projects. A total of 36 microbiologists and 8 epidemiologists from Algeria, Cameroon, Central African Republic, Congo, Congo Democratic Republic, Ivory Coast, Gabon, Madagascar, Mali, Mauritania, Morocco, Niger, Senegal and Tunisia. Benin, Burkina Faso and Chad have been trained. Funding for the training was from WHO, Pasteur Institute of Paris, Centers for Disease Control and Prevention and FAO.

Studies on Salmonella Typhi from nine of countries showed a high proportion of multiresistance in some countries especially in Central Africa and highlight the need to improve surveillance through national laboratories networks. In Cameroon, an outbreak due to Plesiomonas shigelloides was successfully investigated highlighting the importance of collaboration between epidemiologists and microbiologists. Studies conducted in 150 chickens, 90 (60\%) were positive for Salmonella Enteritidis and Hadar $40 \%$ of which were multidrug resistant isolates.

The key challenges are lack of equipment, reagents and inadequate networking and collaboration among laboratories. It is essential, therefore, to establish a network of laboratories through small projects with a view to improving foodborne infections surveillance in particular, data collection and promotion of collaboration between public health, food and animal sectors. A multicentre study on Salmonella infections in human and chicken is also planned. Countries have been provided with reagents and antisera to facilitate serotyping of Salmonella.

The Nigeria project focused on the bacteriological and serological studies on Salmonella serovars in chicken meat in Nigeria. A total of 865 samples were collected from faeces, kidney, lungs, caecum, intestine, liver, heart, gizzard, and cloacal swabs from 525 different chickens. Salmonella was isolated from 130 of the samples and 41 of these were selected for further studies. Thirty-nine out of the 41 samples sero-typed yielded Salmonella Hiduddify; two yielded a rough Salmonella 
serovar. The 39 Salmonella Hiduddify isolates and the two rough isolates were highly similar indicating that all of the isolates were of the same serovar. A low frequency of resistance was found among the isolates, except for resistance to ciprofloxacin for which 23 (56\%) of the isolates tested exhibited resistance [6].

This study by Hendriksen et al. [7] described spread of multidrug resistant Salmonella Concord in Europe and the United States of America among children adopted from Ethiopia. The outcome of this suggests that multi-drug resistant Salmonella Concord clones circulating among Ethiopian adoptees are distinct from other Salmonella Concord clones circulating in Africa. Based on the incident rate of infected Ethiopian adoptees from Denmark, it is estimated that there could potentially be an estimated 52 000 orphans in Ethiopia infected with multidrug resistant Salmonella Concord [7]. Similar findings were reported by Getenet et al. [8].

The study in Mauritius reported the first outbreak of salmonellosis caused by consumption of contaminated marlin mousse [9]. Between 29 October and 5 November 2008, at least 53 persons developed diarrheal illness, all with a history of eating marlin mousse. Salmonella spp. was isolated from stools of 26 affected patients and blood culture from one patient. The same type of Salmonella $s p$ was isolated in three samples of marlin mousse manufactured on 27 October 2008. The constituents of the mousse were smoked marlin, raw eggs, bovine gelatin, oil, and cream. A laboratory investigation of one sample of marlin mousse manufactured 3 days later, and the individual ingredients sampled a week after production of the contaminated batch were all negative for Salmonella. All isolates belonged to Salmonella serovar Typhimurium and were susceptible to all antimicrobials tested [9]. Pulsed-field gel electrophoresis revealed that all the isolates was indistinguishable, thus implicating the mousse as the vehicle of the outbreak [9].

In the Democratic Republic of Congo, Vandenberg et al. [10] evaluated the microbiological and clinical features of Salmonella isolated from children suspected of having bacteremia between 2002 and 2006. A random selection of isolates was typed by pulsed field gel electrophoresis (PFGE). Among the 1528 children included in the study, $26.8 \%$ were bacteremic. Salmonella accounted for $59 \%$ of all bloodstream infections. Salmonella Typhimurium (60.5\%) and Salmonella enteritidis (22.3\%) were the most common Salmonella sero-types. In total, 92.4\% were resistant to at least 3 antimicrobials with the following proportion of strains resistant to: ampicillin (86\%), chloramphenicol (92\%), trimethoprim and sulfamethoxazole (95\%), and tetracycline (34\%). In 2002, 32.1\% of children received an appropriate empiric antimicrobial treatment [10].

These achievements show that bringing microbiologists and epidemiologists from various sectors related to food safety and zoonosis together, provides the opportunity to raise awareness of the individual and common responsibilities of national and international colleagues. Through GFN training activities, national and regional informal networks are created. These interactions with participants during and after training courses results in many interesting issues. Occasionally, these issues are pursued by a small coalition of participants and steering committee members and lead

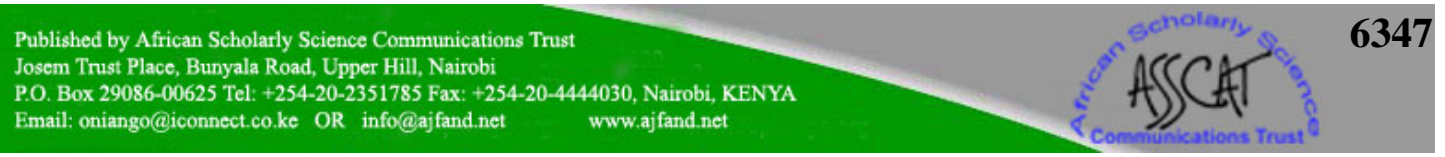


to focused regional or national projects as reported above. By participating in GFN projects, African colleagues team up with GFN core members, exchange ideas and increase their technical capabilities from sample collection, diagnostic and data analysis, to publishing in peer-reviewed international journals. It is important that these results are obtained and published from a region where data usually is considered scarce and fragmented. Participants are also provided with laboratory supplies as well as opportunities to go on study tours in better equipped laboratories to improve on their skills. There is ongoing support for research on AMR in Kenya and several of such projects are planned jointly with other participants (Box 2).

Since 1976, WHO has implemented the Global Environment Monitoring System Food Contamination Monitoring and Assessment Programme (GEMS/Food), which has informed governments, the Codex Alimentarius Commission and other relevant institutions, as well as the public, on levels and trends of contaminants in food, their contribution to total human exposure, and significance with regard to public health and trade. Three scientists from Cameroun, Nigeria and Mali have been trained so far in the WHO African Region on Total Diet studies to estimate the amount of various chemicals associated with the consumption of foods. The institutions to which these scientists are affiliated have been recognized as Centres of Excellence by WHO. Through this programme, a number of countries including Ghana and Mali have conducted studies and submitted data on Persistent Organic Pollutants in breastmilk.

In 2004, WHO launched the International Food Safety Authorities Network (INFOSAN) in collaboration with FAO. The secretariat of INFOSAN is in WHO. The network promotes the rapid exchange of information between Member States during food safety related events; shares information on important food safety related issues of global interest; promote partnership and collaboration between countries, and between networks; and helps countries strengthen their capacity to manage food safety risks. Currently, there are 177 member states, including 33 from countries in Africa. Food safety is an area which requires intersectoral collaboration, thus each country identifies an emergency contact point for the competent authorities responsible for food safety, as well as, focal points from other sectors that play a role in food safety.

INFOSAN shares information on food-related events of international importance through a secure web site. This includes events where people have not become ill but the risk of a health hazard has been identified, in addition to foodborne disease outbreaks. There are many examples where INFOSAN has been used for this purpose including the contamination of infant formula with melamine, thyroid toxicosis related to high iodine in contaminated soy milk, hepatitis A infections linked to internationally traded semi-dried tomatoes and dioxin in pork. INFOSAN also produces information notes to help share lessons learned on events and inform members of important food safety related issues and initiatives. 
Box 2: An integrated surveillance study of antimicrobial resistance in Salmonella spp, Campylobacter spp Escherichia coli and Enterococcus spp from gut of healthy food animals and retail meat outlets in selected regions in Kenya

The main objective of this study is to determine the prevalence and characteristics of antimicrobial resistant Salmonella spp and Campylobacter spp as important foodborne pathogens. The study is also investigating E. coli and Enterococcus spp as important commensal indicator organisms in farm animals that could be reservoirs of antimicrobial resistance gene pools that may be found in human population. The investigations include prevalence of Salmonella spp., Campylobacter spp E. coli and Enterococcus spp from carcass swabs, rectal swabs from cattle, pigs, chickens delivered to 10 main abattoirs in Nairobi (6), Mombasa (2) and Kisumu (2), the three largest cities in Kenya representing central, eastern and western parts of the country, respectively. Meat samples from selected retail outlets in the same regions, and rectal swabs/fecal specimens from cattle, pigs, chickens reared for subsistence and commercial purposes are being sampled along the value chain. In order to understand the interaction of resistance factors between the animal and human populations, isolates of E. coli, Salmonella and Shigella isolated from patients in respective public and private hospitals within the study areas are being tested. For the different settings and animal species that are being studied, the bacterial isolates are being tested for susceptibility to commonly available antimicrobials and investigated for the genetic basis of such resistance.

Data from the study will provide information on prevalence and characteristics of antimicrobial resistance in foodborne pathogens including Salmonella spp, Campylobacter spp, and indicator organisms including E. coli and Enterococcus spp, isolated from farm animals and meat products sold in retail outlets in urban and rural settings in Kenya. The likely interaction and flow of resistance factors within the human population will also be deciphered. These data will form the basis for food safety guidelines for improvement of handling of meat and meat products at abattoirs and retail outlets as well as important baseline for the development of national policies on antimicrobial use and the containment of antimicrobial resistance. Data collection is well advanced and the findings will be published soon.

\section{Challenges and Opportunities for Foodborne Disease Surveillance}

The coming into force of the IHR (2005), revision of the IDSR, which includes indicators for foodborne disease surveillance, as well the adoption of various global and regional resolutions on food safety which includes charges for stretching of foodborne diseases surveillance provide opportunities for strengthening the surveillance systems in countries including foodborne disease surveillance. These 
have increased awareness for food safety and the importance of surveillance in food control resulting in modest steps for systems strengthening.

An analysis of food control systems has so far been carried out in 26 countries between 2008 and 2010. Food laboratories have improved substantially as compared to the status in 2002 when the previous situation analysis was conducted. There are several laboratories conducting various microbiological and chemical tests in the countries that have completed the situation analysis. Three main challenges were identified and these are: lack of equipment and human resources; underutilization of certain laboratories and lack of synergy among laboratories. The general directive is to use detailed audits of the laboratories by identifying their strengths and weaknesses. These laboratories could be strengthened and assigned new mandates for foodborne disease surveillance and food monitoring rather than building new structures.

The bringing together of microbiologists and epidemiologists from various sectors related to food safety and zoonosis through the GFN activities provides the opportunity to raise awareness of the individual and common responsibilities of national and international colleagues. The GFN training activities offers opportunities for formation of informal national and regional networks and ensures follow-up of participants' activities outside the training cycle. These offer participants the opportunity to participate in the laboratory quality assurance program such as External Quality Assurance System (EQAS), taking advantage of routine Subtyping facilities including serotyping, phage typing, antibiotic resistance profiling and molecular typing, e.g., pulsed-field gel electrophoresis (PFGE).

Despite these efforts, commitment to food safety and foodborne disease surveillance in particular still remains low, resulting in inadequate resources both human and financial. Most food safety programmes and food safety systems remains fragmented resulting in duplication of efforts and inefficient use of resources. There are challenges with data reporting, for example, 33 countries have registered to report data to the GFN country data bank but only $11^{1}$ have focal points that can submit data. So far only Senegal has regularly submitted data while Mali and South Africa have submitted data once.

\section{The Next Steps}

In order to strengthen foodborne disease surveillance as an integral part of integrated diseases surveillance it is imperative that WHO continues supporting countries to strengthen their food safety systems including laboratories, foodborne disease surveillance and provision of the policy and legal basis for food safety and strengthening partnerships for surveillance activities including cooperation between the public and private sectors and between medical, food and veterinary microbiologists and epidemiologists. It is essential to strengthen cooperation among

\footnotetext{
${ }^{1}$ Eritrea, Ethiopia, Gambia, Ghana, Mali, Mauritius, Mozambique, Nigeria, Rwanda, Senegal, South Africa 
government agencies for investigation, control and policy and promoting research for early detection of outbreaks. More resources should be allocated for food safety including foodborne disease surveillance; strengthening of laboratory and other systems; and setting public health goals for countries to monitor progress with surveillance data.

Joint capacity building efforts with IHR and other programmes should continue by conducting courses to augment and strengthen laboratory-based surveillance and outbreak detection and response skills in microbiology and epidemiology. Innovative methods including supply of reagents and antisera; mentoring of selected participants in the GFN programmes; collaboration with selected trainees to carry out specific work; research; and on site training of selected individual should be intensified. Countries should also take advantage of regional and global networks like GFN, WHO Collaborating Centres, Regional centres; External Quality Assurance System (EQAS) of WHO GFN; Country data bank, a secure website for countries to submit data and Electronic list serve which is electronic mailing list that distributes messages to members. There is a need for full participation of African countries in foodborne disease surveillance activities. This includes ensuring that countries nominate emergency contact points and focal points who will play an active role. They should also have good communication with the national IHR focal points. Regular risk mitigation and risk communication using WHO approved methods including risk analysis, HACCP and the WHO Five Keys to Safer Foods is essential. 


\section{REFERENCES}

1. Nyikal J, Misore A, Nzioka C, Njuguna C, Muchiri E, Njau J, Maingi S, Njoroge J, Mutiso J, Onteri J, Langat A, Kilei IK, Nyamongo J, Ogana G, Muture B, Tukei P, Onyango C, Ochieng W, Tetteh C, Likimani S, Nguku P, Galgalo T, Kibet S, Manya A, Dahiye A, Mwihia J, Mugoya I, Onsongo J, Ngindu A, DeCock KM, Lindblade K, Slutsker L, Amornkul P, Rosen D, Feiken D, Thomas T, Mensah P, Eseko N, Nejjar A, Onsongo M, Kessel F, Njapau H, Park DL, Lewis L, Luber G, Rogers H, Backer L, Rubin C, Gieseker KE, Azziz-Baumgartner E, Chege W and A Bowen Outbreak of Aflatoxin Poisoning - Eastern and Central Provinces, Kenya, January - July 2004, MMWR Weekly 2004; 53(34); 790-793.

2. Azziz-Baumgartner E, Kimberly L, Gieseker K, Heather Strosnider HH, Azziz-Baumgartner, Banziger EM, Bhat RV, Breiman R, Brune M-N, DeCock K, Dilley A, Groopman J, Hell K, Henry SH, Jeffers D, Jolly C, Jolly P, Kibata GN, Lewis L, Liu X, Luber G, McCoy L, Mensah P, Miraglia M, Misore A, Njapau H, Ong C-N, Onsongo MTK, Page SW, Park D, Patel M, Phillips T, Pineiro M, Pronczuk J, Rogers SH, Rubin C, Sabino M, Schaafsma A, Shephard G, Stroka J, Wild C, Williams JT and D Wilson Workgroup Report: Public Health Strategies for Reducing Aflatoxin Exposure in Developing Countries. Environ Health Perspect. 2006; 114(12): 1898-1903.

3. WHO. Outbreak of bromide intoxication in Cacuaco Municipality, Angola. Report of WHO Support Mission, Nov 21 to Dec 5, 2007.

4. WHO. Health Regulation (2005) WHO, Geneva 2006.

5. Tauxe RV Surveillance and investigation of foodborne diseases; roles for public health in meeting objectives for food safety. Food Control 2002; 13:363-369.

6. Raufu I, Hendriksen RS, Ameh JA and FM Aarestru Occurrence and characterization of Salmonella Hiduddify from chickens and poultry meat in Nigeria. Foodborne Pathog Dis. 2009; 6(4):425-30.

7. Hendriksen RS, Mikoleit M, Kornschober C, Rickert RL, Duyne SV, Kjelsø C, Hasman H, Cormican M, Mevius D, Threlfall J, Angulo FJ and FM Aarestrup Emergence of multidrug-resistant Salmonella Concord infections in Europe and the United States in children adopted from Ethiopia, 2003-2007. Pediatr Infect Dis J. 2009 Sep;28(9):814-8.

8. Beyene G, Nair S, Asrat D, Mengistu Y, Engers $\mathbf{H}$ and $\mathbf{J}$ Wain Multidrug resistant Salmonella Concord is a major cause of salmonellosis in children in Ethiopia, J Infect Dev Ctries 2011; 5(1):023-033. 
9. Issack MI, Hendriksen RS, Lun PL, Lutchun RK and FM Aarestrup Salmonella enterica serovar Typhimurium in Mauritius linked to consumption of marlin mousse. Foodborne Pathog Dis. 2009 Jul-Aug;6(6):739-41.

10. Vandenberg O, Nyarukweba DZ, Ndeba PM, Hendriksen RS, Barzilay EJ, Schirvel C, Bisimwa BB, Collard JM, Aidara Kane A and FM Aarestrup Microbiologic and clinical features of Salmonella species isolated from bacteremic children in eastern Democratic Republic of Congo. Pediatr Infect Dis J. 2010 Jun;29(6):504-10. 